\title{
Experiencias de Arte Latinoamericano y su incidencia sobre el tiempo: Producción, mediación y difusión de mensajes Proyecto de Investigación \\ $\mathrm{N}^{\circ} 11.1$
}

Equipo de Investigación ${ }^{(1)}$

Natalia Aguerre

por Facultad de Diseño y Comunicación, Universidad de Palermo (ARG),

Marie Boivent

por Universite Rennes 2 (FRA)

\begin{abstract}
Resumen: El Proyecto de Investigación 11.1 reflexiona acerca de la relación de las manifestaciones estéticas con la temporalidad y la memoria. Realiza aportes significativos sobre esta temática mediante el estudio de relatos orales, escritos, plásticos, teatrales, performáticos, y/o cinematográficos, abordando la pluralidad del tiempo y la construcción de la memoria para la transformación del mapa de lo perceptible y de lo pensable, en torno al arte y la comunicación.
\end{abstract}

Palabras clave: Arte - Temporalidades - Memoria.

[Resúmenes en inglés y portugués en la página 88]

${ }^{(1)}$ Los CVs del Equipo de Investigación pueden consultarse en el Capitulo Directores de Líneas y Coordinadores de Proyectos de esta misma Edición.

\section{Acerca del Proyecto 11.1}

Experiencias de Arte Latinoamericano y su incidencia sobre el tiempo:

Producción, mediación y difusión de mensajes

El Proyecto 11.1 Experiencias de Arte Latinoamericano y su incidencia sobre el tiempo: Producción, mediación y difusión de mensajes, reúne una serie de reflexiones en las que se conjugan temporalidades y expresiones de la memoria producto de los procesos históricos, políticos, culturales, de las innovaciones y del devenir de las tecnologías de la comunicación. Este último aspecto es sustancial, dado que las nuevas plataformas digitales no 
solo permiten la producción y circulación de diferentes formas y modos de construcción de la memoria, de creación y experimentación de la contemporaneidad.

Se acordó entre la Universidad de Palermo y la Universite Rennes 2 (Francia), avanzar en una investigación en la que participan académicos de ambas Instituciones, con la coordinación compartida de Natalia Aguerre (UP) y Marie Boivent (Universite Rennes 2, Francia), con el fin de generar un debate acerca de cómo las prácticas artísticas entrecruzan tramas temporales mediante lenguajes expresivos y diferentes medios propiciando nuevas matrices narrativas y/o reconstruyendo la memoria colectiva.

Las reflexiones y los resultados obtenidos en el Proyecto 11. 1 Experiencias de Arte Latinoamericano y su incidencia sobre el tiempo: Producción, mediación y difusión de mensajes se continúan desarrollando en el Proyecto 11.2 Arte, Historia y Memoria, en el marco de la misma Línea de Investigación y bajo la misma Directora Natalia Aguerre.

Sus principales objetivos son:

- Generar un espacio de participación crítica y producción de conocimiento, en torno a los procesos y prácticas artísticas desde la perspectiva comunicacional.

- Abordar la cuestión del arte desde la mirada comunicacional y en relación a la Innovación estética, medios y nuevos lenguajes.

- Establecer diálogos y contrapuntos entre dichas áreas y sus problemáticas con el campo comunicacional.

- Intervenir productivamente desde la comunicación sobre el universo del arte repensando sus modos de producción, mediación y difusión de mensajes.

- Experimentar de modo activo las sensibilidades expresivas para poder abordar el análisis y la crítica de los distintos lenguajes comunicacionales.

- Establecer el lugar político, social, cultural y económico de los análisis estéticocomunicacionales.

- Estimar las dimensiones de las estructuras productivas, de sus campos expresivos y comunicacionales en relación a los contextos sociopolíticos y culturales, nacionales, internacionales y globales.

\section{Acerca de la Línea 11}

Arte y Comunicación dirigida por Natalia Aguerre se desarrolla de manera ininterrumpida desde 2015 en la Facultad de Diseño y Comunicación (UP, Argentina), e incluye hasta el momento cuatro proyectos finalizados el 11.1 Experiencias del Arte Latinoamericano y su incidencia sobre el tiempo coordinado por Natalia Aguerre (UP, Argentina) y Marie Boivent (Universite Rennes 2, Francia), el 11.2 Arte, Historia y Memoria coordinado por Natalia Aguerre (UP, Argentina) y Carlos Paz (Universidade do Vale dos Sinos, Unisinos, Rio Grande do Sul, Brasil), el 11.3 Arte y Diseño: Discursos de la Identidad Cultural en América Latina coordinado por Natalia Aguerre (UP, Argentina) y Cynthia Lizette Hurtado Espinosa (Universidad de Guadalajara, México), y el 11.4 Imagen e Identidad Política en América Latina coordinado por Marina Mendoza por (UP, Conicet IEALC UBA, Ar- 
gentina), Mariano Dagatti (Conicet, Universidad de Quilmes, Argentina) y Paulo Carlos López López (Universidad de Santiago de Compostela, España).

\section{Mapa de Áreas y Proyectos}

El Proyecto 11.1 se vincula con todas las carreras de la Facultad de Diseño y Comunicación puesto que los lineamientos abordados son transversales a los contenidos relacionados con el diseño en sus múltiples manifestaciones, y particularmente con la carrera de Arte Multimedial. Asimismo, sus resultados tienen una capacidad de impacto en las carreras de posgrado que exploran las interrelaciones entre arte, comunicación y diseño en toda la región.

\section{Productos y Resultados}

\section{a)- Publicaciones}

Cuaderno del Centro de Estudios de Diseño y Comunicación No75. (2019) Arte y Comunicación: Experiencias estéticas y el flujo del tiempo. Coordinación Natalia Aguerre y Marie Boivent. Año XX, Diciembre 2019, Buenos Aires, Argentina. ISSN: 1668-0227. Esta publicación documenta y comunica los resultados alcanzados en el proyecto de investigación 11.1 Experiencias del Arte Latinoamericano y su incidencia sobre el tiempo, y a continuación se detallan los autores y artículos contenidos en ella:

Aguerre, Natalia; Boivent, Marie (2019) Prólogo (Pp. 13-19)

Capasso, Verónica (2019) Nuevas tramas socio-espaciales después de la inundación en la ciudad de La Plata: un análisis de experiencias artísticas y memoria colectiva (Pp. 21 a 35)

Cisneros, Julia (2019) Operaciones de montaje y reescritura como huellas del tiempo en "Diagonal Cero" (Pp. 37 a 50)

de la Cruz Lichet, Virginia (2019) Vers une taxonomie de la mémoire. Pratiques artistiques colombiennes sur la reconstitution de faits historiques (Pp. 51-66)

Forero Hurtado, Andrea del Pilar; Orozco, Yulieth Aldana; Rodríguez Páez, Luis Carlos (2019) El presente y el irremediable pasado. La reconstrucción de lo público desde la música rap de la Alianza Urbana en Quibdó-Chocó, Colombia (Pp. 67-81) Fajole, Florent (2019) Mirtha Dermisache : Lécriture autre, à elle-même (Pp. 83-97) García Aranguren, Erick (2019) Vanguardias artísticas y videojuegos: retomar el pasado para el mercado futuro (Pp. 99-116)

Garaglia, Laura (2019) "Cómo hacer palabras con cosas" (Pp. 117-128)

Gómez, Lía (2019) El cine y esos pueblitos: Mediaciones culturales de la memoria nacional (Pp. 129-140)

Gustavino, Berenice (2019 Avant-garde, dépendance culturelle et périodisations en lutte. L'historicisation de l’art argentin des années 1960 (Pp. 141-151) 
Jaubet, Franco (2019) Poesía de lo real en "Historia de un Clan" de Luis Ortega (Pp. 153-164)

Juárez, Camila; Lamilla, Julio (2019) Prácticas sonoras desbordantes. El surgimiento del ciclo Experimenta97 en Buenos Aires (Pp. 165-180)

Mihal, Ivana; Matarrese, Marina (2019) Diversidad cultural y pueblos indígenas: una mirada sobre las TIC (Pp. 181-193)

Paz, Carlos D. (2019) De esta suerte se gobierna la mayor parte. La jefatura indígena examinada desde la intencionalidad performativa de la escritura etnológica de la C (Pp. 195-211)

Torres, Margarita Eva (2019) Tiempos de Amor (Pp. 213-225)

Vallina, Carlos; Vallina, Cecilia (2019) Imagen y Memoria (Pp. 227-237)

Actas de Diseño No27. (2019) XIII Encuentro Latinoamericano de Diseño "Diseño en Palermo" IX Congreso Latinoamericano de Enseñanza del Diseño Comunicaciones Académicas. Facultad de Diseño y Comunicación, Universidad de Palermo. Buenos Aires, Argentina. Se documentan las ponencias de la Comisión Arte y Comunicación: Proyecto 11.1 Experiencias del Arte Latinoamericano y su incidencia sobre el tiempo correspondientes a la presentación del Cuaderno 75 durante el III Coloquio de Investigación y Desarrollo en Diseño (Ponencias en Pp. 43-44).

\section{b)- Congresos / Coloquios / Plenarios}

III Coloquio de Investigación y Desarrollo en Diseño Latino. Universidad de Palermo, 30 de julio de 2018. IX Congreso Latinoamericano de Enseñanza del Diseño. Semana Internacional del Diseño en Palermo.

En la comisión Investigar en Diseño se presentaron las reflexiones y conclusiones del proyecto 11.1 Experiencias del Arte Latinoamericano y su incidencia sobre el tiempo. El volumen Cuadernos No75 fue destinado a reflexionar acerca de la relación de las manifestaciones estéticas con el tiempo y la memoria. El arte es un campo donde se conjugan temporalidades y expresiones de la memoria producto de los procesos históricos, políticos, culturales, de las innovaciones estéticas y del desarrollo de las tecnologías. Este último aspecto es sustancial en nuestro presente si consideramos la Internet como un medio que permite el abordaje de diferentes formas y modos de construcción de la memoria, de creación y experimentación de la contemporaneidad; es decir, de re-presentar con el flujo del tiempo. Se centró en debatir cómo las prácticas artísticas de nuestra región entrecruzan tramas temporales mediante lenguajes expresivos y diferentes medios propiciando nuevas matrices narrativas y/o reconstruyendo la memoria colectiva.

Fundados en la necesidad de hacer crecer los alcances de la producción artística regional y argentina, y teniendo en cuenta los cambios que se están produciendo a partir de la revolución tecnológica digital, se presentaron artículos inéditos que analicen en clave crítica y desde distintas perspectivas los procesos que involucran estos temas. El propósito fue reflexionar acerca de cómo las prácticas artísticas latinoamericanas construyen y vinculan temporalidades divergentes a través de tejidos de discursos -históricos, políticos, tecnológicos y estéticos-, donde encuentran asiento para la simbolización y la formula- 
ción de diferentes estados de representación cultural; en definitiva para llevar adelante una operación de creación y transmisión comunicativa. Cuadernos №75 procura ahondar y realizar aportes significativos sobre esta temática mediante el estudio de relatos orales, escritos, imaginarios pictóricos, teatrales, performáticos, televisivos y cinematográficos, fusionando el pasado con el presente para la transformación del mapa de lo perceptible y de lo pensable, en torno al arte y la comunicación. Expusieron: Verónica Capasso, Julia Cisneros, Laura Garaglia, Lía Gomez, Franco Jaubet, Camila Juarez, Julio Lamilla y Margarita Torres.

A continuación se detallan las ponencias presentadas en esta Comisión coordinada por la Directora del proyecto Natalia Aguerre:

Verónica Capasso

Arte y memoria después de la inundación. La configuración de nuevas tramas socioespaciales en La Plata

Julia Cisneros

Operaciones de montaje y reescritura como huellas del tiempo en Diagonal Cero Laura Garaglia

¿Cómo hacer palabras con cosas?

Lia Gómez

El cine y esos pueblitos: mediaciones culturales de la memoria nacional

Franco Jaubet

Poesía de lo real en "Historia de un Clan" de Luis Ortega

Camila Juarez, Julio Lamilla

Prácticas sonoras desbordantes. El surgimiento del ciclo Experimenta 97 en Buenos Aires

Margarita Torres

Tiempos de Amor

$1^{\circ}$ Plenario de Directores de Investigación DC, 13 de octubre - 2017. Es este primer plenario los Directores de Líneas y Proyectos de Investigación presentaron a sus pares y al conjunto del Programa de Investigación de la Facultad de Diseño y Comunicación, los resultados obtenidos y/o en proceso (publicaciones y acuerdos), junto con los Proyectos. La Directora de la Línea de Investigación $\mathrm{N}^{\circ} 11$ presento a sus pares, los principales avances del Proyecto 11.1 Experiencias del Arte Latinoamericano y su incidencia sobre el tiempo.

\section{c)- Formación de Posgrado e Impacto curricular}

La Directora Natalia Aguerre es es Docente de Posgrado en Diseño de la Facultad de Diseño y Comunicación: Doctorado en Diseño y Maestría en Gestión del Diseño y al igual que Marie Boivent (Universite Rennes 2, Francia) en su institución, incorporan los contenidos de su investigación a sus asignaturas en posgrado. 


\title{
Evaluación Externa
}

El Proyecto 11.1 Experiencias de Arte Latinoamericano y su incidencia sobre el tiempo: Producción, mediación y difusión de mensajes, con la Universite Rennes 2 (Francia), cuyos resultados fueron publicados en el Cuaderno No75, fue evaluado exitosamente por los Evaluadores Amanda López y Roberto Londoño en 2019.

\begin{abstract}
Research Project 11.1 reflects on the relationship of aesthetic manifestations with temporality and memory. Makes significant contributions on this topic through the study of oral, written, plastic, theatrical, performative, and / or cinematographic stories, addressing the plurality of time and the construction of memory for the transformation of the map of the perceptible and the thinkable, around art and communication.
\end{abstract}

Keywords: Art - Temporalities - Memory

Resumo: O Projeto de Pesquisa 11.1 reflete sobre a relação das manifestações estéticas com a temporalidade e a memória. Fazer contribuições significativas sobre este tema através do estudo de histórias orais, escritas, plásticas, teatrais, performativas e / ou cinematográficas, abordando a pluralidade do tempo e a construção da memória para a transformação do mapa do perceptível e do pensável, em torno da arte e da comunicação.

Palavras chave: Arte - Temporalidades - Memória

[Las traducciones de los resúmenes fueron realizadas a través de traductor automático] 